\title{
Análisis Didáctico del proceso matemático de Modelización en alumnos de Secundaria
}

\author{
Martín M. Socas, Universidad de La Laguna (España) \\ Raquel M. ${ }^{a}$ Ruano, Universidad de La Laguna (España) \\ Josefa Hernández, Universidad de La Laguna (España)
}

Recibido el 5 de enero de 2016; aceptado el 16 de enero de 2016.

\section{Análisis Didáctico del proceso matemático de Modelización en alumnos de Secundaria}

\section{Resumen}

En este trabajo se analiza didácticamente el proceso matemático de Modelización en relación con las dificultades y errores de un grupo de alumnos de Educación Secundaria. Se consideran dos situaciones problemáticas relativas a este proceso, que se estudian desde las perspectivas epistemológica, semiótica y fenomenológica que propone el Enfoque Lógico Semiótico (ELOS) (Socas, 2007). Se concluye relacionando estas dificultades y errores de los alumnos con la organización de la enseñanza de la Modelización en la Secundaria, mostrando también el papel que juegan en dicho proceso otros como la Sustitución Formal y la Generalización, así como las estructuras y las operaciones matemáticas implicadas en el mismo.

Palabras clave. Análisis Didáctico; Modelización Matemática; Educación Secundaria; Enfoque Lógico Semiótico (ELOS); Dificultades y Errores.

\section{Análise Didática do processo de Modelagem matemática em estudantes de Secundário}

\section{Resumo}

Neste trabalho analisa didaticamente o processo matemático de Modelagem em relação às dificuldades e erros de um grupo de estudantes de Educação secundária. Duas situações-problema em relação a este processo serão analisadas a partir da perspectiva epistemológica, semiótica e fenomenológica, que propõe a aproximação Lógica Semiótica (ALS) (Socas, 2007). Conclui-se relacionando essas dificuldades e os erros dos estudantes com a organização do ensino de modelagem na Secundária, mostrando o papel de outros processos como a Substituição Formal e a Generalização, como tambén as estruturas e os operações matemáticos neste processo.

Palavras chave. Análise Didática; Modelagem Matemática; Educação Secundária; Aproximação Lógica Semiótica (ALS); Dificuldades e erros.

Para citar: Socas, M., Ruano, M.R., Hernández, J. (2016). Análisis Didáctico del proceso matemático de Modelización en alumnos de Secundaria. Avances de Investigación en Educación Matemática, 9, 21 -

41.

(C) Sociedad Española de Investigación en Educación Matemática (SEIEM). www.seiem.es 


\section{A Didactic Analysis of the Mathematical Modelling Process in Secondary School Students}

\section{Abstract}

This work didactically analyzes the mathematical modelling process in terms of the difficulties and errors of a group of secondary school students. Two problem situations concerning this process are considered here, which are studied from the epistemological, semiotic and phenomenological perspective proposed in the Semiotics Logical Approach (SLA) (Socas, 2007). The work concludes by relating these difficulties and errors of students with the organization of the teaching of modelling in secondary school, also showing the role played by Formal Substitution and Generalization, as well as structures and mathematical operations involved in the process itself.

Key words. Didactic Analysis; Mathematical Modelling; Secondary Education; Semiotic Logical Approach (SLA); Difficulties and Errors.

\section{Analyse Didactique du processus mathématique de Modélisation chez des élèves de Secondaire}

\section{Résumé}

À ce travail le processus mathématique de Modélisation est didactiquement analysé en relation avec les difficultés et les erreurs d'un groupe d'élèves d'Education Secondaire. Deux situations problématiques relatives à ce processus seront examinés depuis les perspectives epistemologique, semiotique et fenomenologique qui propose l'Approche Logique Semiotique (ALS) (Socas, 2007). Il conclut en rapportant ces difficultés et les erreurs des élèves à l'organisation de l'enseignement de la Modélisation à l'école secondaire, montrant aussi le rôle que d'autres processus jouent comme la Substitution Formelle et la Généralisation, ainsi que les structures et les opérations mathématiques dans le dit processus.

Paroles clés. Analyse Didactique; Modélisation Mathématique; Education Secondaire; Approche Logique Semiótique (ALS); Difficultés et Erreurs.

\section{Introducción}

El Análisis Didáctico del proceso matemático de Modelización se desarrollará desde el marco competencial que propone el Enfoque Lógico Semiótico (ELOS) (Socas, 2007). En este Enfoque se considera la cultura matemática como un proceso de "culturización matemática", es decir, de producción del conocimiento en esta cultura. Se distinguen y estudian los tres aspectos esenciales que caracterizan la producción de este conocimiento: el Epistemológico, considerado desde la perspectiva de los objetos y de los métodos del campo conceptual tratado; el Semiótico, que supone la denotación de los objetos matemáticos a través de las representaciones, en las que el lenguaje natural y las representaciones analógicas, digitales y virtuales juegan un papel relevante en la significación y funcionalidad de los objetos matemáticos considerados, y el Fenomenológico, como lo que puede percibirse en una situación problemática de los objetos, los métodos y las representaciones matemáticas. Aspectos que tienen que ser necesariamente considerados en el proceso de matematización de la cultura en el Sistema Educativo. De esta manera, la Modelización Matemática se caracteriza fenomenológicamente como el proceso matemático por excelencia, en el que se necesita determinar los elementos matemáticos básicos (objetos), así como las diferentes relaciones que éstos tienen entre sí. 
La Modelización se considera, en este Enfoque, como un proceso esencial de las Matemáticas que se transfiere, en general, a las diferentes ramas del conocimiento, y se trata en la Educación Matemática como un objeto matemático de referencia.

Se presentan distintas perspectivas a nivel internacional que dan lugar a diferentes enfoques, tanto sobre la aplicación de la modelación en el aula, como a su uso en la investigación, con el objetivo de situar la Modelización Matemática en ELOS en este contexto internacional. Se requiere para ello analizar, brevemente, los diferentes supuestos básicos del enfoque competencial: Análisis Didáctico, Competencia Matemática Formal, Competencia Cognitiva y Competencia de Enseñanza, que propone ELOS, en particular los dos primeros que se utilizarán en este trabajo de investigación.

La presentación de este trabajo se organizará en torno a los siguientes apartados:

- Análisis Didáctico, que se describe junto con la Competencia Matemática Formal.

- El proceso de Modelización Matemática, que se analiza desde la Cultura Matemática y desde la Educación Matemática y se sitúa en el modelo de Competencia Matemática Formal, describiéndose desde las tres perspectivas: epistemológica, semiótica y fenomenológica.

- Una propuesta de investigación sobre dificultades y errores de los alumnos de Secundaria en el proceso matemático de Modelización.

- Análisis del contenido de dos situaciones problemáticas sobre Modelización Matemática desde el punto de vista competencial, que se considerarán, en el apartado siguiente, para analizar y clasificar los errores cometidos por los alumnos en relación con dicho proceso.

- Discusión de los resultados obtenidos en el análisis de las respuestas dadas a las dos preguntas anteriores sobre el proceso de Modelización por alumnos de Secundaria. Dificultades y errores.

- Consideraciones finales, en las que se describen, especialmente, desde el punto de vista educativo el planteamiento y el desarrollo del proceso de Modelización Matemática con alumnos de Educación Secundaria.

\section{Análisis Didáctico}

Se comienza con una descripción breve de algunos supuestos básicos del Enfoque Lógico Semiótico (ELOS) (Socas 2007 y 2010), entendido como una propuesta teórico-práctica (formal-experimental) que pretende aportar instrumentos para el análisis, la descripción y la gestión de las situaciones problemáticas o fenómenos de naturaleza didáctica matemática que ocurren en el microsistema educativo, a efectos de caracterizar otro supuesto básico de este enfoque: el Análisis Didáctico.

Los aspectos lógicos y semióticos de ELOS tienen como referencia la Fenomenología de Peirce (1987). La Semiótica, que se deriva de esta Fenomenología, es una teoría de la realidad y del conocimiento que podemos tener de los fenómenos por el único medio que disponemos: los Signos (considerados como un Metalenguaje que permite conocer la realidad). En el análisis del Signo surge la inferencia semiótica que Peirce construye como una Teoría Lógica, es decir, la Semiótica, entendida como un proceso lógico a la vez formal (análisis) y material (proceso de inferencia). En esta 
inferencia semiótica lo que se analizan son las marcas o expresiones observables y ostensibles de la inferencia, que Pierce organiza como una teoría lógica que tiene tres referentes estrechamente ligados entre sí que llamó: Primero, Segundo y Tercero.

Por ello, si pretendemos estudiar cualquier fenómeno (situación problemática), que es el punto de partida en ELOS, éste se analizará, siempre, a partir de un contexto determinado y mediante tres referentes organizados como primero, segundo y tercero, que se expresan mediante una representación triangular que describe las relaciones entre los tres referentes.

ELOS planifica y gestiona la investigación de los problemas didácticos matemáticos que se dan en el microsistema educativo mediante el uso de cuatro supuestos básicos que denomina: Competencia Matemática Formal, Competencia Cognitiva, Competencia de Enseñanza y Análisis Didáctico.

El modelo de Competencia Matemática Formal (CMF) explicita lo que queremos significar por la complejidad de los objetos, tanto los conceptos, relaciones y procedimientos, como el conjunto de situaciones y fenómenos, que pueden ser analizados mediante la organización lógico-formal de los objetos matemáticos implicados. Se apoya, fundamentalmente, en los campos numérico, algebraico y analítico y tiene como referente las tres componentes del campo conceptual: operaciones, estructuras y procesos, y como contexto: las situaciones problemáticas, el lenguaje (representaciones) y los argumentos (razonamientos).

En él se expresan los diferentes dominios de la actividad matemática en relación con el campo conceptual, desde la perspectiva formal y sus diferentes relaciones, es decir, permite describir los objetos del campo conceptual en términos epistemológicos, semióticos y fenomenológicos (Socas, 2010).

El Modelo de Competencia Cognitivo es el segundo referente de la semiosis, y tiene en cuenta los aspectos anteriores (modelo de Competencia Matemática Formal, primer referente de la semiosis). Se refiere a las funciones cognitivas específicas de los objetos matemáticos tratados y a los aspectos estructurales del aprendizaje, es decir, simulará los procesos cognitivos implicados en la ejecución competente de un usuario ideal del campo conceptual considerado.

El Modelo de Competencia de Enseñanza es el tercer referente de la semiosis, y, considera, igualmente, los aspectos anteriores (modelos Competencia Matemática Formal, primer referente, y Cognitivo, segundo referente) y describe las acciones de los sujetos implicados, los procesos de comunicación, los mediadores, las situaciones, los contextos..., que se dan en la enseñanza (Socas, 2007). En este sentido ELOS necesita establecer un modelo de competencia que permita identificar y comprender el Problema Didáctico Matemático que se quiere tratar en el microsistema educativo. La construcción de este modelo de competencia, denominado Análisis Didáctico, toma como marco de referencia los organizadores del currículo (Rico, 1997) y la noción inicial que planteó Freudhental (1983) para el análisis didáctico, como "el análisis de los contenidos curriculares de las matemáticas que se realiza al servicio de la organización de su enseñanza en los sistemas educativos" (Puig, 1997).

El Análisis Didáctico, en ELOS, queda organizado mediante la siguiente triada: Matemáticas curriculares, analizadas desde la Competencia Matemática Formal, representaciones semióticas y dificultades, obstáculos y errores (Figura 1). 


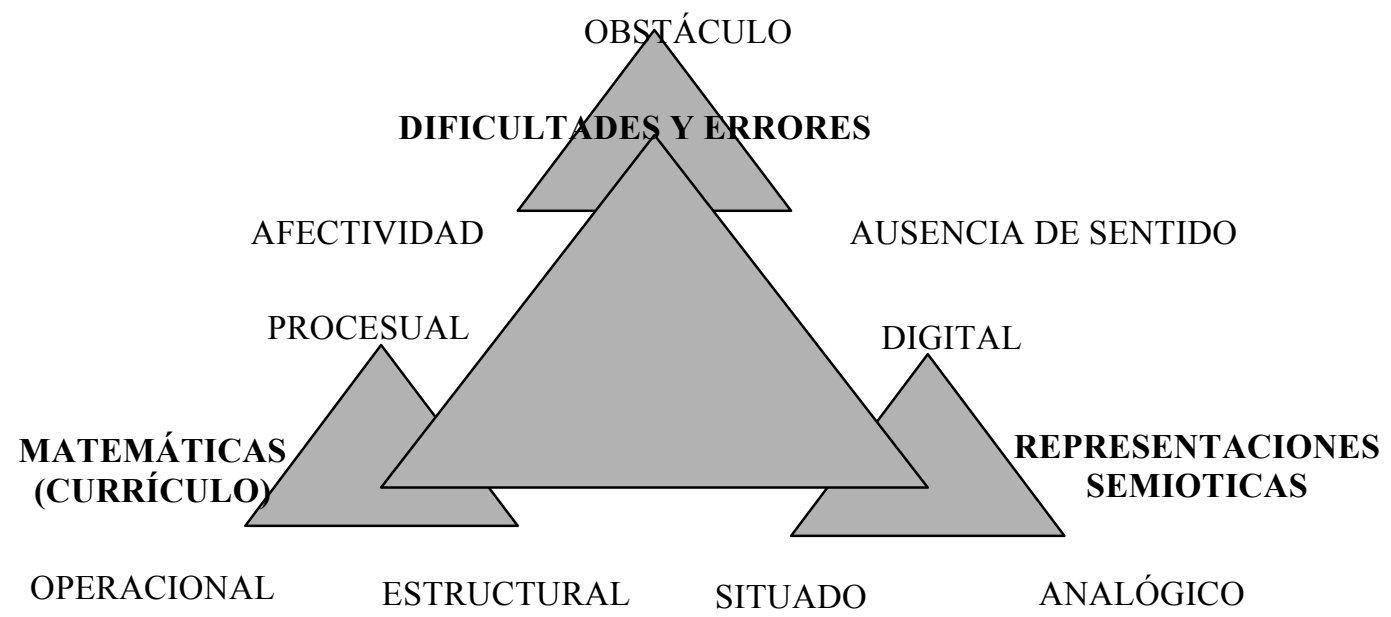

Figura 1. Análisis Didáctico en ELOS.

El Análisis Didáctico implica, en relación con la componente currículo, una revisión de los contenidos curriculares desde la perspectiva formal: operacional, estructural y procesual; es decir, se describe el campo conceptual de los objetos involucrados, así como las funciones y fenómenos que queremos que organice en este nivel educativo.

La componente representaciones semióticas implica una revisión de los contenidos curriculares en relación con las diferentes formas de representación de los objetos, así como la presentación de la información al alumnado; en este apartado, se pueden considerar los diferentes estadios de desarrollo del objeto tratado: semiótico, estructural y autónomo.

La componente dificultades y errores supone una revisión de los contenidos curriculares en relación con estos tres aspectos, con una doble finalidad de prevención y remedial, lo que permitirá, por ejemplo, desde la perspectiva de prevención establecer los niveles o competencias cognitivas requeridas de los alumnos, en relación con el objeto o foco matemático tratado. La determinación de los errores que generan los alumnos necesita de elementos de análisis que permitan profundizar en la complejidad de las dificultades del aprendizaje de las Matemáticas. Una manera de abordarlos sería, como señala Socas (1997), tomar en consideración las tres direcciones de análisis, a modo de tres ejes de coordenadas que nos situaría con más precisión en los orígenes del error y nos permitiría como profesores, arbitrar procedimientos y remedios más efectivos. Estos tres ejes estarían determinados por su origen: I) En un obstáculo; II) En ausencia de sentido; III) En actitudes afectivas y emocionales.

El Análisis Didáctico es, en consecuencia, una propuesta de organización que permite la identificación del problema y la comprensión del mismo, bien sea un problema de organización curricular o un problema de investigación didáctico matemático de enseñanza y aprendizaje.

En este trabajo, el Análisis Didáctico facilita la identificación y comprensión del problema didáctico matemático que queremos estudiar: las dificultades y errores de alumnos de Secundaria resolviendo dos situaciones problemáticas de Modelización. Y 
se desarrolla, especialmente, en la relación "Currículo de Matemáticas" con "Dificultades y Errores", considerando el objeto matemático Modelización en dichas situaciones. No se analizan de manera específica las Representaciones y sus estadios de desarrollo, sin embargo, es obvio que su presencia tiene que ser considerada en relación al proceso de Sustitución Formal, presente siempre en cualquier Modelización Matemática.

Conviene, finalmente, resaltar que el constructo Análisis Didáctico es también un supuesto básico fundamental en otros enfoques, aunque, en general, todos parten de la descripción inicial de Freudhental (1983), mencionada anteriormente. Especial reconocimiento requiere el Análisis Didáctico en Educación Matemática como metodología de investigación, formación de profesores e innovación curricular, desarrollado por los miembros del Grupo de investigación: "Didáctica de la Matemática. Pensamiento Numérico" de la Universidad de Granada, en el Plan Andaluz de Investigación, Desarrollo e Innovación (Rico, Lupiáñez y Molina, 2013). El Análisis Didáctico lo organizan en cinco categorías, que tienen un carácter cíclico, y que denominan Análisis Conceptual, del Contenido, Cognitivo, de Instrucción y Evaluativo. Categorías tomadas de los organizadores del currículo, cerrando el análisis desarrollado en cada una con una necesaria síntesis, que denominan, respectivamente: Conceptos y red de significados que articulan un tema, Focos prioritarios, Organización de los aprendizajes, Diseño de la unidad didáctica y Revisión del proceso (Rico y Fernández Cano, 2013, p.19). Se trata de una propuesta de investigación que se desarrolla en un proceso cíclico de análisis-síntesis y que integra los resultados obtenidos a priori y a posteriori.

El Análisis Didáctico que se propone en esta investigación, también puede ser abordado desde este enfoque con las cuatro primeras categorías.

\section{EI Proceso de Modelización Matemática}

La modelización ha sido un foco de atención para los investigadores en los últimos 40 años. Durante las últimas reformas curriculares de inicios del siglo XXI se ha introducido la Modelización en los currículos de un gran número de países. Parece imponerse así un acuerdo social, reforzado por programas internacionales como PISA (Programme for International Student Assessment) a nivel preuniversitario y los desarrollos del pacto de Bolonia para la enseñanza superior, sobre la conveniencia de introducir en todos los niveles de enseñanza aspectos relacionados con las aplicaciones de las Matemáticas y con la Modelización (Burkhardt, 2006).

Desde 2005, la Sociedad Europea de Investigación en Educación Matemática, ERME, creó un grupo específico sobre el tema liderado por la investigadora alemana Gabriele Kaiser que, a su vez, es la editora de dos números especiales de la revista internacional Zentralblatt für Didaktik der Mathematik ZDM dedicados exclusivamente al mismo.

En España, también hay un gran interés por la enseñanza de la Modelización, como se expresa en la revista UNO. Didáctica de las Matemáticas, que publicó en 2002 un número especial sobre "Modelización y matemáticas".

Este interés relacionado con las aplicaciones de la Modelización Matemática (MM) en Educación Matemática ha llegado a consolidarse como un campo de investigación en esta disciplina científica (Blum et al., 2007), que se ha denominado, en general, Modelación Matemática; de esa forma, el uso de la modelación en la escuela se 
muestra de diferentes maneras según los puntos de vista desde donde se mire la didáctica y de acuerdo a los objetivos de la actividad. Actualmente hay estudios con enfoques muy variados, Kaiser y Sriraman (2006) presentan una clasificación de los trabajos, atendiendo a criterios de tipo epistemológico y a otros aspectos propios de la Educación Matemática.

En nuestro caso, la Modelización Matemática se sitúa en el modelo Competencia Matemática Formal (CMF) (Socas, 2010 y 2012), uno de los Modelos de Competencia que conforman el Enfoque Lógico Semiótico (ELOS) (Socas, 2007), y en él se describe la Modelización como uno de los tres procesos fundamentales que caracterizan los campos conceptuales numérico, algebraico y analítico, junto con la Sustitución Formal y la Generalización.

Desde el punto de vista matemático, el planteamiento de una situación problemática que involucre el proceso de Modelización, se puede organizar a partir de situaciones diversas de naturaleza intramatemática o extramatemática, en las que se parte de una descripción no organizada de un comportamiento regular en la que no está explicitada la regla.

A partir de la situación problemática o de la formulación de la tarea, ésta se puede describir mediante cinco fases, que facilitan el planteamiento y la resolución y que la caracterizan matemáticamente: (1) Sistematización, explicitación y reconocimiento de la regla; (2) Matematización o formulación en términos de la regla; (3) Resolución en términos de la regla, mediante la representación elegida, lo que comporta el análisis del modelo construido; (4) Validación (verificación) de la regla; y (5) Interpretación.

Resumidamente, la descripción de la Modelización Matemática, desde la Disciplina Matemática, se realiza al considerar los diferentes aspectos que caracterizan este objeto matemático: epistemológicos, semióticos y fenomenológicos, es decir, describe la Modelización Matemática en el campo conceptual y el contexto, así como determina las relaciones existentes.

El Enfoque Lógico Semiótico (ELOS) se sitúa, inicialmente, en la perspectiva epistemológica, al igual que el enfoque de la Educación Matemática Realista (EMR) o de la Teoría Antropológica de lo Didáctico (TAD) (Kaiser y Sriraman, 2006), sin embargo, propone que la Modelización Matemática, no debe ser considerada, únicamente, desde la visión epistemológica, sino que hay que tener en cuenta también las perspectivas semiótica y fenomenológica.

Por ejemplo, en Chevallard (1989), se introduce un esquema simplificado de la actividad o proceso de modelización elemental en el cual intervienen esencialmente dos tipos de entidades: un sistema matemático o extramatemático y un modelo (matemático) de este sistema. Según el autor, el proceso de modelización queda descrito en las siguientes tres etapas: (1) describimos el sistema que queremos estudiar, (2) construimos el modelo propiamente dicho y (3) implementamos el modelo obtenido.

En ELOS, el proceso de Modelización Matemática también supone el desarrollo de las tres etapas anteriores que se desarrollan, en la práctica educativa, en las cinco fases descritas anteriormente, y que no son necesariamente lineales.

\section{Una propuesta de investigación sobre dificultades y errores de los alumnos de Secundaria en el proceso matemático de Modelización}


Este artículo sobre la Modelización Matemática es parte de un estudio sobre los tres procesos fundamentales de la Cultura Matemática: Sustitución Formal, Generalización y Modelización, cuyos objetivos generales son: (1) Estudiar la competencia matemática de los alumnos cuando desarrollan tareas relacionadas con los procesos de Sustitución Formal, Generalización y Modelización en Álgebra y (2) Estudiar los errores que cometen los alumnos en tareas relacionadas con los citados procesos atendiendo especialmente a su origen. En esta presentación trataremos aspectos del segundo objetivo centrándonos en el proceso de Modelización.

Para responder a los objetivos de investigación, se diseñó un cuestionario con 15 preguntas y 43 ítems, en torno a los procesos: Sustitución Formal, Generalización y Modelización, que posibilitó el análisis y clasificación de los errores, así como conjeturar el origen de los mismos. Para indagar en la validez de estas conjeturas, se realizaron entrevistas clínicas.

El diseño y modo de construcción del cuestionario se recogen en Ruano y Socas (2001). El cuestionario se administró en dos partes, C1 y C2, que contienen cada una 3 cuestiones, relativas a la Modelización.

La población de estudio estuvo constituida por 60 estudiantes de Educación Secundaria (4. ${ }^{\circ}$ de ESO y $1 .^{\circ}$ de Bachillerato) del IES Puerto de la Cruz, (Puerto de la Cruz) y del IES San Matías (La Laguna), ambos en Tenerife.

\section{Análisis del contenido de dos situaciones problemáticas sobre Modelización Matemática desde el punto de vista competencial}

Una de las tareas básicas del profesor de Matemáticas es organizar el contenido matemático para enseñarlo, lo que supone un problema profesional que requiere el análisis, la comprensión y la planificación. El primer ámbito que necesita planificar es el contenido matemático que deriva de la propia disciplina, que podemos denominar contenido matemático disciplinar o formal.

El modelo de Competencia Matemática Formal (CMF), descrito anteriormente, es un instrumento útil que, teniendo en cuenta el contexto, permite relacionar y describir tanto a nivel individual como institucional los significados de los objetos matemáticos y sus relaciones, los errores, las dificultades y los obstáculos.

El CMF es un instrumento técnico que puede ayudar a los profesores a analizar los significados atribuidos a los objetos matemáticos desde la perspectiva institucional (currículo y libros de texto, entre otros), pero también se puede utilizar para caracterizar el dominio de la actividad matemática, en las diferentes tareas que se propongan al alumnado y, en consecuencia, analizar e interpretar sus producciones en la realización de estas tareas, así como los errores y hacer conjeturas sobre las dificultades, obstáculos y el origen de los mismos, tanto desde el punto de vista de la docencia como del de la investigación.

Consideramos dos situaciones problemáticas relacionadas con la Modelización Matemática, extraídas del cuestionario 2 de la Memoria de Tesis: "Competencias y errores de alumnos de Secundaria en los procesos de Sustitución Formal, Generalización y Modelización. Implicaciones didácticas para la transición del lenguaje aritmético al algebraico", que está en fase de redacción para su presentación y lectura. El cuestionario está basado en los tres procesos fundamentales relacionados con la Cultura Matemática: Sustitución Formal, Generalización y Modelización, 
aunque en este trabajo estudiaremos, a modo de ejemplo, las preguntas 4 y 6 del cuestionario 2, relacionadas con la Modelización.

La pregunta 4 propone una situación problemática situada en el conocimiento procesual, particularmente, en el proceso de Modelización, en la que se pide la identificación y resolución de la misma. Estas tareas persiguen el desarrollo de las competencias generales de todo proceso matemático: reconocerlo, formularlo $\mathrm{y}$ manipularlo que, en este caso, se concretan en las fases o momentos que caracterizan el proceso de Modelización.

PREGUNTA 4: Una persona tiene un terreno rectangular de dimensiones 12 metros de frente y 8 metros de fondo. Después, esa misma persona, compra un terreno contiguo de 64 metros cuadrados. Una segunda persona le propone cambiar su terreno completo por otro rectangular, en la misma calle, con la misma área y el mismo fondo, pero en mejor sitio.

¿Cuánto debe medir el frente del nuevo terreno para que el trato sea justo?

Se trata de una situación problemática en la que figuran enunciados verbales, dados en lenguaje natural, dentro de un contexto geométrico. Podemos distinguir tres situaciones:

- Los resolutores pueden interpretar la situación problemática como una modelización geométrica-numérica, en la que realizando una Sustitución Formal (conversión de registro al lenguaje gráfico o numérico), considerando las estructuras geométricas implícitas en el enunciado de la tarea (área, longitud,...), utilizan una técnica para determinar el resultado mediante operaciones.

- Los resolutores pueden interpretar la situación problemática como una generalización algebraica, para lo cual deben ser capaces de identificar los datos, las incógnitas y las relaciones existentes entre ellos, y plantear para todos los rectángulos de área $8 \cdot \mathrm{x}$, la ecuación adecuada para el que tiene de área $64 \mathrm{~m}^{2}$, esto es: $160=8 \cdot \mathrm{x}$, siendo x la longitud del frente del nuevo terreno. En este caso, atenderemos a las estrategias utilizadas para la resolución de la ecuación (tanteo, procedimientos aritméticos, algebraicos o la combinación de varios) y a la comprobación (validación) de que el resultado satisface las condiciones del problema.

- Los resolutores pueden dar un paso más allá e interpretar la situación planteada como una modelización funcional, en la que realizan una generalización, por entender que la situación planteada es una particularización del caso general $f(x)=8 x$, que relaciona el área de todos los rectángulos de ancho 8 metros y largo desconocido.

Los argumentos o razonamientos considerados son los relacionados con los esquemas partes-todo, las inducciones o las deducciones realizadas. La pregunta puede presentar dificultades para los alumnos ya que su correcta resolución exige el dominio de conceptos asociados a la Geometría: superficie, área, dimensiones, longitud, largo, ancho y, por lo tanto, el reconocimiento de la estructura geométrica implícita en el problema.

PREGUNTA 6: Una batalla de la conquista de Canarias.

Guanches, con sus hondas y piedras, contra ingleses, con sus arcabuces. La "efectividad" de los ingleses es de 1/5, es decir, 1 de cada 5 ingleses alcanza a herir a un guanche (cada 5 minutos). La efectividad de los guanches es de 1/20 (la cuarta parte de la efectividad inglesa).

a) ¿Cuánto tiempo tardaría la batalla si hubiera 200 guanches y 100 ingleses? 
b) ¿Cuánto tiempo debería tardar la batalla si se desconoce el número de guanches e ingleses? ¿Quiénes deben ganar la batalla?

En la pregunta 6, los alumnos también deben encontrar un modelo para el problema citado. En principio presenta mayor dificultad que el anterior, porque el contexto elegido no es tan conocido por los alumnos como el del terreno. Además, en esta actividad existe la dificultad añadida de la utilización de fracciones.

Esta situación se presenta en un contexto general. Se pretende analizar el comportamiento de los alumnos, en relación con el hecho de fijar o no el número de participantes, para dar respuesta a los diferentes ítems. Análogamente a la pregunta anterior, consideraremos el ítem (6.a) regular, si interpreta y encuentra las relaciones entre las variables aunque no llegue a obtener el modelo final.

Se consideran las respuestas dadas por los alumnos a estas dos preguntas para analizar y clasificar los errores cometidos por los alumnos en el proceso de Modelización que estamos estudiando.

\section{Discusión de los resultados obtenidos a partir del análisis de las respuestas dadas a las dos preguntas anteriores sobre el proceso de Modelización por alumnos de Secundaria. Dificultades y errores}

En este apartado se analizan los errores cometidos por los alumnos en las dos preguntas cuyo análisis del contenido se expuso anteriormente y se conjetura el origen de los errores. El análisis de los errores se hará siguiendo el modelo propuesto en Socas (1997 y 2007).

La clasificación de los errores se realiza mediante diferentes esquemas de análisis. En las preguntas con respuestas abiertas empleamos una adaptación de las redes sistémicas de Bliss y otros (1983), con modificaciones para ajustarlas a nuestro propósito. En concreto, en el lado derecho de la red, hemos incluido un recuadro con el número del alumno que ha recorrido cada itinerario. La inclusión de este código nos permite hacer un seguimiento y comprobar si en cada alumno existe una persistencia en el mismo tipo de error o, por el contrario, varía dependiendo de la tarea presentada.

De manera general, en un primer análisis de las respuestas dadas a las seis preguntas relacionadas con la Modelización en el cuestionario, se observa que tienen grandes dificultades con el proceso de Modelización. Así, de los seis ítems dedicados a este proceso, tres no han obtenido ninguna respuesta correcta, siendo $23,74 \%$ el porcentaje medio de aciertos en preguntas relacionadas con el mismo, los porcentajes de aciertos dependen de las tareas realizadas y son, en general, muy dispares. Los mayores porcentajes de aciertos se encuentran en el reconocimiento del modelo, con una media del 36,37\% y, los menores, en la validación del modelo con un 13,64\% de aciertos. La fase de formulación y desarrollo del modelo se sitúa en medio en cuanto a dificultad, con un $21,21 \%$ de aciertos.

Consideramos, en primer lugar, los errores concretos cometidos por los alumnos en la pregunta 4 del Cuestionario C2, cuyo análisis del contenido realizamos antes. Dado que esta pregunta admite una respuesta abierta, antes de tratar de organizar los errores, se introduce una red sistémica, que presentamos como Red 1 (Figura 2). 


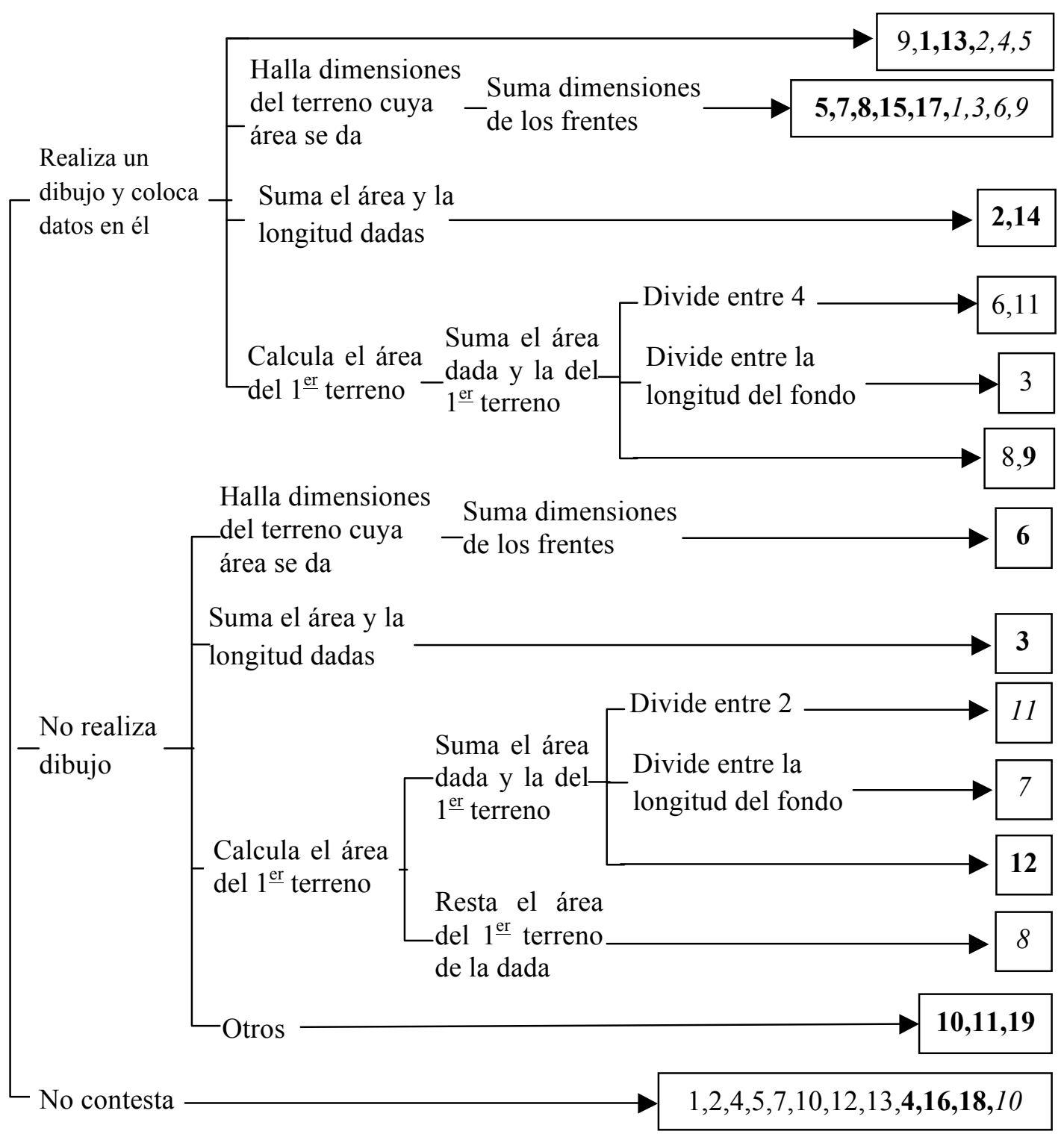

Figura 2. Red 1. Errores de los alumnos en la pregunta 4.

Esta red nos ayuda a clarificar los procesos de resolución seguidos por los alumnos y observar, por ejemplo, que el mayor número de errores ha sido dejar incompleta la resolución del problema y que un número considerable de errores se ha producido porque sólo representan gráficamente la situación planteada, pues aun siendo capaces de realizar la conversión entre el lenguaje natural y el gráfico, no han sabido interpretarla para poder responder a la pregunta.

Observamos, también, en la Red 1, que los alumnos han seguido tres razonamientos diferenciados:

1. Dividen el área del terreno dado entre 2 y posteriormente lo suman a la longitud dada.

2. Calculan el área del nuevo terreno y posteriormente la dividen entre 2 .

3. Calculan el área del nuevo terreno y posteriormente la dividen entre 4. 
Esta organización de los errores en la Red 1 permite obtener información relevante, pero se necesita clasificar los errores, para lo que construimos el Esquema siguiente (Figura 3).

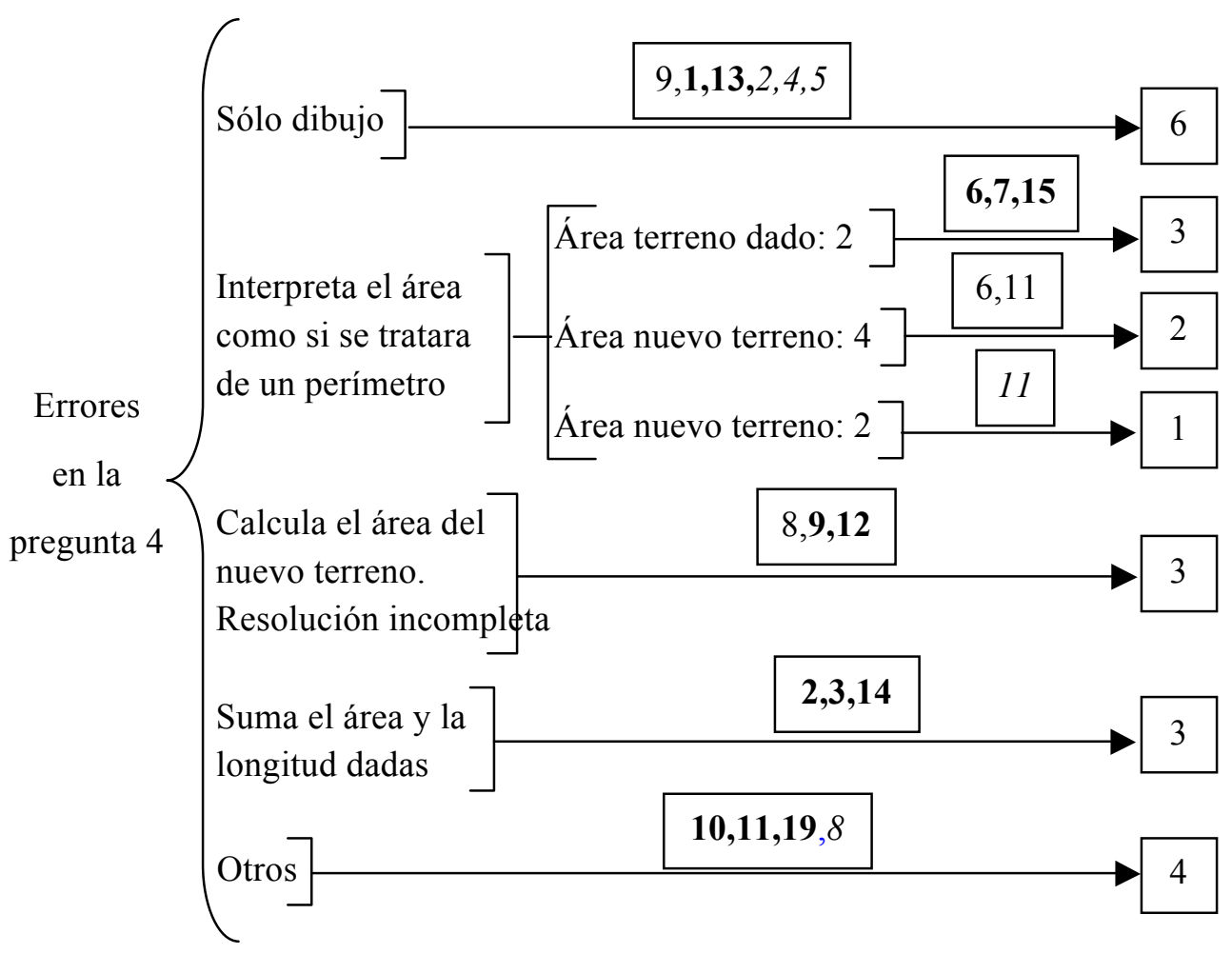

Figura 3. Esquema de los errores en la pregunta 4.

Además de las observaciones anteriores, se puede inferir que los alumnos no tienen clara la realización de cambios de registro y conjeturamos que su origen es la ausencia de sentido, debido a las características propias del Álgebra. Otro grupo de alumnos ha obtenido el área del nuevo terreno correctamente, pero no ha continuado para alcanzar la solución de la situación problemática planteada. Aunque todos los pasos seguidos por estos alumnos en el proceso de resolución del problema son correctos, parece que no tienen claro que lo que se les pide es el frente del terreno, no su área. Este error puede deberse a que no saben cómo continuar la resolución, en cuyo caso, situamos su origen en una ausencia de sentido debida a las características propias del Álgebra o bien, a que confunden lo que es la longitud con el área, en cuyo caso, situamos el origen del error en una ausencia de sentido con origen en la Geometría o simplemente, no se sienten motivados para terminar el problema, en cuyo caso, situamos el origen del error en las actitudes afectivas.

Otro error es la confusión entre longitud y área, los alumnos suman el área dada con la longitud del frente del primer terreno. Suponemos que este tipo de error tiene su origen en una ausencia de sentido debido a que los alumnos no han terminado de comprender la diferencia entre los conceptos geométricos de área y longitud. Otro grupo de alumnos interpreta el área como si se tratara del perímetro del rectángulo, pues para hallar la longitud del frente dividen el valor del área entre el número de lados de la figura. 
En relación con los razonamientos diferenciados en la Red 1, podemos concretar que en este caso:

- Dividen el área del terreno dado entre 2 y, posteriormente, lo suman a la longitud dada.

El método de resolución es correcto, pero confunden la raíz cuadrada con la división entre 2. Consideramos que este tipo de error tiene su origen en una ausencia de sentido, debida a dificultades que han quedado sin resolver en la Aritmética.

- Calculan el área del nuevo terreno y, posteriormente, la dividen entre dos.

El método de resolución es diferente al usado en el razonamiento anterior, pero el error es el mismo, es decir, confunden la raíz cuadrada con la división entre 2.

- Calculan el área del nuevo terreno y, posteriormente, la dividen entre 4.

Éste es el caso más claro de confusión del perímetro con el área, pues para hallar la longitud de uno de los lados del rectángulo, dividen el área entre el número de lados de dicha figura.

Por último, hay alumnos que no siguen un proceso de resolución claro, éstos están englobados en la categoría "otros". Por ejemplo, un alumno emplea de forma errónea una regla de tres para resolver el problema. Otro calcula el área del primer terreno pero luego, en vez de sumarla con el área dada, la resta, concluyendo que la longitud del nuevo frente debe ser $32 \mathrm{~m}^{2}$.

En el Esquema (Figura 4) se resumen los orígenes de los errores.

Figura 4: Esquema de los errores en la pregunta 4.

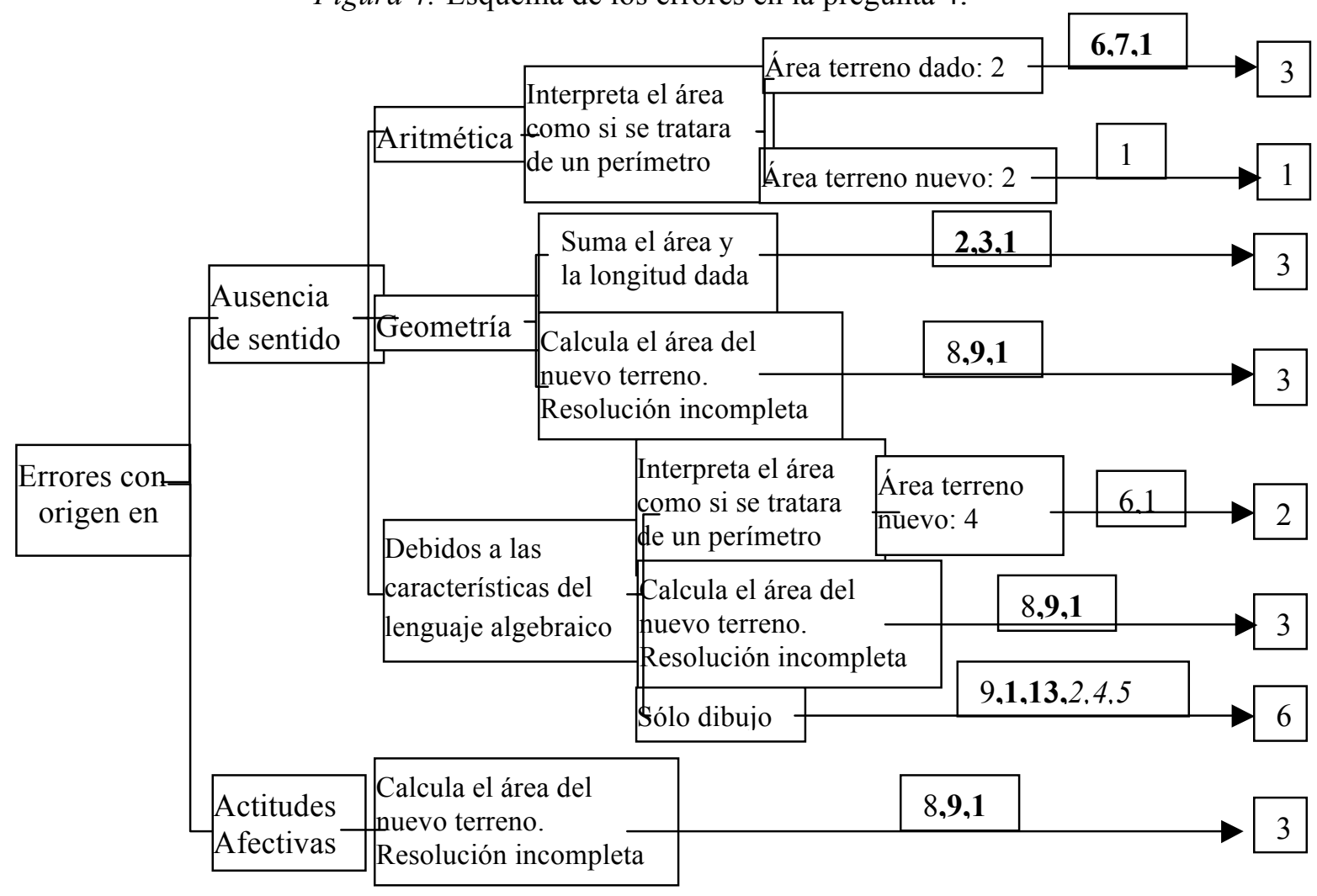


Consideramos, a continuación, los errores cometidos por los alumnos en la pregunta 6 del Cuestionario C2, que se recogen en el siguiente esquema (Figura 5).

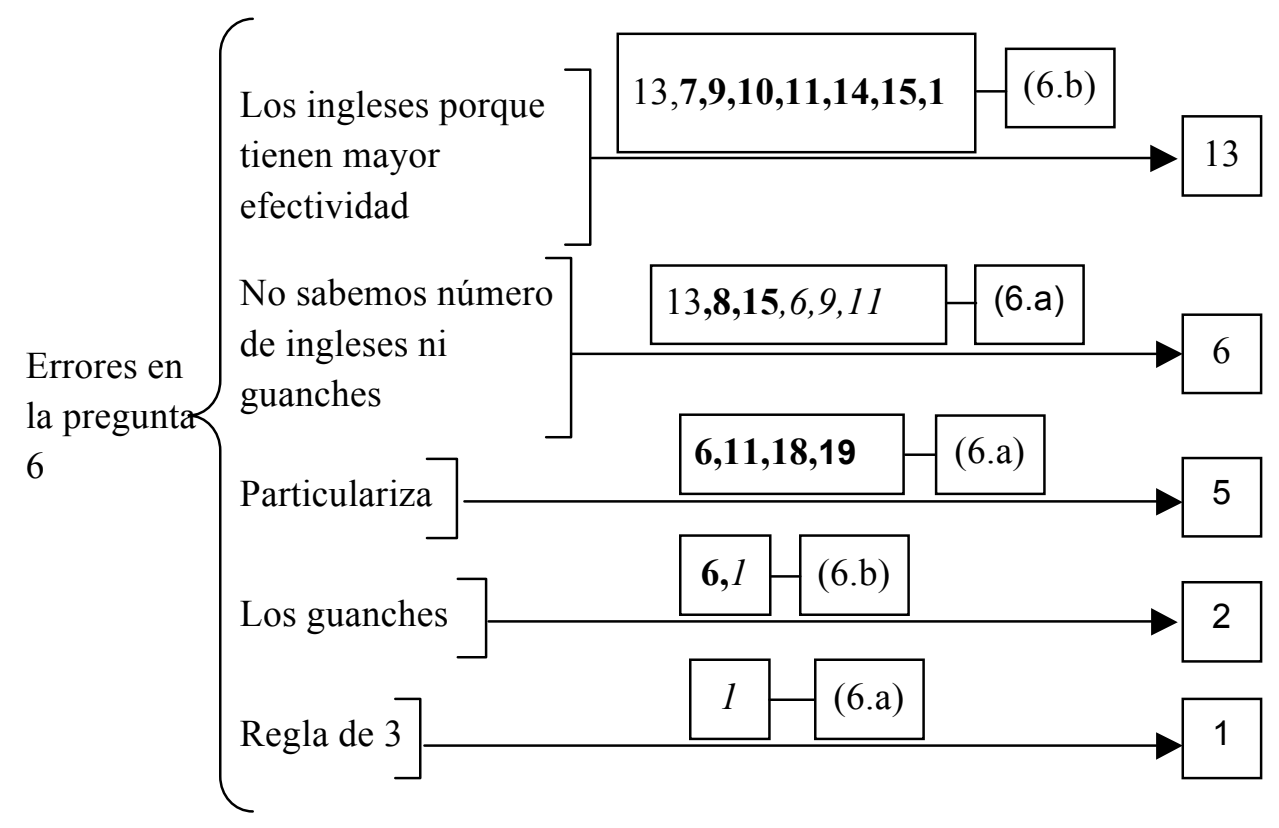

Figura 5: Esquema de los errores en la pregunta 6.

Como se observa en el esquema precedente, el fallo más repetido, es considerar que los ganadores de la batalla serán los ingleses debido a su mayor efectividad. Esto representa que ha habido un análisis parcial de las variables que intervienen en el modelo, estos alumnos no han reparado en que el número de combatientes es una variable a tener en cuenta. La mayoría de los alumnos han respondido sin buscar una razón lógica, simplemente por contestar algo. No han sido lo suficientemente persistentes o simplemente no han encontrado el problema interesante como para buscar una estrategia para resolverlo. Del mismo modo han procedido aquellos que han contestado que los ganadores debían ser los guanches sin ningún tipo de fundamentación. Así, en ambos casos, se considera que el origen del error está en las actitudes afectivas y emocionales hacia las Matemáticas. Otro de los fallos cometidos es considerar que el problema no se puede resolver porque no sabemos ni el número de ingleses ni el de guanches. Muchos alumnos dieron este tipo de respuesta en el problema anterior, y como en ese caso, pensamos que el origen del error está en una ausencia de sentido con origen en la Aritmética. Estos alumnos no ven la necesidad de utilización de las letras para representar las variables desconocidas, y como no se les da un valor numérico consideran que les faltan datos para resolver el problema. Un tercer tipo de error es el de particularización, éste se ha venido repitiendo en casi todas las preguntas y, como hemos reiterado, significa que el alumno permanece estancado en un pensamiento numérico. Este estancamiento también podría deducirse del anterior tipo de error, la no utilización de las letras cuando son necesarias. Del mismo modo que hemos hecho en ocasiones anteriores, situamos el origen de este error en una 
ausencia de sentido ocasionada por problemas que han quedado sin resolver en la Aritmética.

Pasamos a organizar los errores atendiendo a su origen en el siguiente esquema (Figura 6).

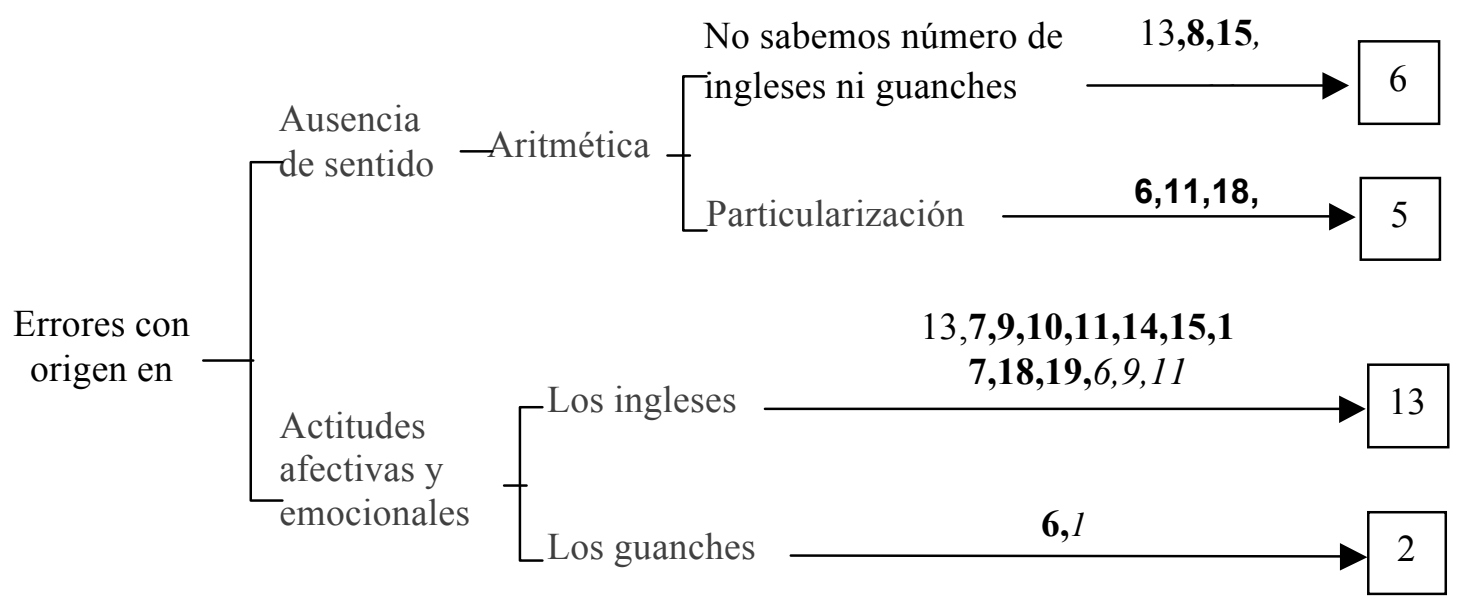

Figura 6: Esquema de los errores en la pregunta 6.

Finalmente, organizaremos los errores cometidos por los alumnos en las preguntas relativas al proceso de Modelización. En general, se pueden situar en relación a tres orígenes distintos: actitudes afectivas hacia las Matemáticas, ausencia de sentido y obstáculo, aunque, debemos señalar, que en el proceso de Modelización no hemos encontrado ningún error con origen en obstáculos.

En la siguiente tabla (Tabla 1) se recogen los errores en todas las preguntas relacionadas con el proceso de Modelización. En la 1. ${ }^{a}$ columna aparece el posible origen del error, en la segunda el campo conceptual, y en la $3 .{ }^{\mathrm{a}}$, la denominación de cada tipo de error.

Tabla 1. Errores en la Modelización.

\begin{tabular}{|c|c|c|}
\hline Origen & $\begin{array}{l}\text { Campo } \\
\text { conceptual }\end{array}$ & Tipo de error \\
\hline \multirow{3}{*}{$\begin{array}{l}\text { Actitudes } \\
\text { afectivas }\end{array}$} & & Resolución incompleta \\
\hline & & Modelo incompleto \\
\hline & & $\begin{array}{c}\text { Análisis parcial de las variables del modelo (Guanches o } \\
\text { ingleses) }\end{array}$ \\
\hline \multirow{9}{*}{$\begin{array}{l}\text { Ausencia de } \\
\quad \text { sentido }\end{array}$} & \multirow{6}{*}{ Aritmética } & $\begin{array}{l}\text { Interpreta el área como si fuera un perímetro } \\
\text {-Confusión de raíz cuadrada y división entre } 2\end{array}$ \\
\hline & & Uso incorrecto de la regla de tres \\
\hline & & Particularización \\
\hline & & Confusión entre multiplicación y potencia \\
\hline & & Faltan datos \\
\hline & & No sabemos número de guanches ni ingleses \\
\hline & \multirow{3}{*}{ Geometría } & $\begin{array}{c}\text { Interpreta el área como si fuera un perímetro (Divide } \\
\text { entre } 4 \text { el área del terreno) }\end{array}$ \\
\hline & & Resolución incompleta \\
\hline & & Confusión entre longitud y área \\
\hline
\end{tabular}




\begin{tabular}{|c|c|c|}
\hline & \multirow{3}{*}{$\begin{array}{c}\text { Características } \\
\text { propias del } \\
\text { lenguaje } \\
\text { algebraico } \\
\end{array}$} & Sólo dibujo \\
\hline & & Resolución incompleta \\
\hline & & Cambio de registro incorrecto \\
\hline
\end{tabular}

\section{Consideraciones finales}

Desde el punto de vista educativo, el planteamiento de una situación problemática que involucre el proceso de Modelización Matemática (MM), se puede organizar a partir de situaciones diversas: físicas, económicas, numéricas, algebraicas, analíticas, geométricas..., en las que se parte de una descripción no organizada de un comportamiento regular en la que no está explicitada la regla.

A partir de una situación problemática o de la formulación de la tarea, que supone su identificación o reconocimiento, el citado proceso se puede describir mediante cinco fases que facilitan el planteamiento y la resolución y que la caracterizan matemáticamente: (1) Sistematización, explicitación y reconocimiento de la regla; (2) Matematización o formulación en términos de la regla; (3) Resolución en términos de la regla, mediante la representación elegida, lo que comporta el análisis del modelo construido; (4) Validación (verificación) de la regla y, (5) Interpretación.

En resumen, la caracterización de la MM como objeto matemático de la Disciplina Matemática, se debe realizar tomando en consideración los diferentes aspectos que caracterizan a cualquier objeto matemático: epistemológicos, semióticos y fenomenológicos, es decir, describir la MM en el campo conceptual y en el contexto, así como determinar las relaciones existentes.

Desde ELOS, al igual que desde la Educación Matemática Realista (EMR) o desde la Teoría Antropológica de lo Didáctico (TAD), se considera la Modelización Matemática de sistemas matemáticos, esto es, la modelización intramatemática como, por ejemplo, la modelización algebraica de un sistema numérico o topológico y la modelización diferencial de un sistema geométrico, como una parte esencial de la actividad de modelización que es inseparable de la modelización de sistemas extramatemáticos. En efecto, aunque el proceso de modelización parte de un sistema extramatemático, por ejemplo, un sistema proveniente de las ciencias de la salud, de las ciencias económicas o de las ciencias físicas como sistema que se desea modelizar, el progresivo desarrollo de la actividad de modelización incluye, necesariamente, etapas en las que interviene la modelización intramatemática.

Esta noción de MM es coherente con el desarrollo histórico de las Matemáticas y permite considerarla como un proceso de matematización progresiva de un sistema en el cual el primer modelo pasa a jugar el papel de sistema (matemático) y así sucesivamente, lo que conduce a trabajar con "modelos de modelos" del sistema inicial. Aparece así, claramente, el carácter recursivo de la actividad de Modelización Matemática.

Entendemos que si ésta excluye los sistemas intramatemáticos, el proceso de estudio de un sistema extramatemático queda abortado a partir de la primera modelización, lo que limita la noción de modelización.

Proponemos, pues, una forma de interpretar la Modelización Matemática que incluye de manera esencial la modelización matemática de sistemas matemáticos, esto es, aquellos procesos que se llevan a cabo para responder a cuestiones problemáticas 
que surgen en un sistema matemático (como, por ejemplo, un sistema aritmético, geométrico, entre otros) cuya resolución requiere la construcción de un modelo matemático (que puede ser algebraico, analítico o de cualquier otro tipo) como un trabajo en dicho modelo.

Asumimos que la modelización no es únicamente un aspecto de las Matemáticas, sino que toda actividad matemática puede ser interpretada como una actividad de modelización. En toda actividad matemática se puede identificar un sistema en torno al cual se formulan cuestiones problemáticas que motivan y dan origen a la construcción de ciertos modelos.

De esta manera, se considera, el proceso de Modelización como un proceso matemático que requiere del control constante del modelo en la situación problemática inicial, es decir, de su adecuación o no, y de su capacidad para dar respuesta tanto a las cuestiones iniciales como a las que van apareciendo a lo largo del proceso.

Si analizamos, también, la caracterización fenomenológica que se propone en ELOS para los campos conceptuales numéricos, algebraicos y analíticos, observamos que la modelización es el proceso cumbre que puede implicar a todos los objetos del campo conceptual; en particular, a los procesos de Generalización y Sustitución Formal, así como a las Estructuras y a las Operaciones. Sin embargo, esto no significa que toda $\mathrm{MM}$ suponga necesariamente la implicación de todos los objetos matemáticos, sino que esta se puede determinar por diferentes caminos. Estos caminos o rutas permiten caracterizar fenomenológicamente la $\mathrm{MM}$ en el campo conceptual considerado, mediante el uso del Modelo de Competencia Matemática Formal (CMF). De esta manera tenemos que la MM está determinada, en el camino más corto, por: Modelización - Sustitución Formal - Técnicas Operatorias - Operaciones y, en el camino más largo, por: Modelización - Generalización - Sustitución Formal Estructuras - Operaciones.

En cualquier caso, todos los caminos están contextualizados en las Situaciones problemáticas, en las Representaciones y en los Argumentos (Razonamientos) que están implicados en el desarrollo de las Situaciones Problemáticas.

Finalmente, en relación con los tres Procesos (Sustitución Formal, Generalización y Modelización), conviene señalar que estos responden a las competencias matemáticas generales propios de cada proceso, que son: el reconocimiento del proceso, su formulación y su manipulación (validación), como señala Chevallard (1989) para el proceso de MM.

En resumen, desde el punto de vista educativo, debemos tener en consideración que todo proceso de MM conlleva un proceso de Sustitución Formal, es decir, supone siempre el uso de, al menos, dos representaciones (lenguaje natural, algebraico, geométrico...), por lo que resulta necesario realizar la conversión entre las distintas representaciones y las transformaciones en ellas.

En la práctica educativa, la mayor parte de las situaciones problemáticas, que implican el proceso de MM, también necesitan del proceso de Generalización algebraica, lo que requiere el uso de varias tareas relacionadas con el proceso de Generalización; entre ellas la particularización, la iteración, la recursividad, el método inductivo... En este sentido, podemos considerar el proceso de MM como un proceso recursivo, al aplicar tareas que caracterizan a la Generalización algebraica. Igualmente, a la hora de validar el modelo y comprobar si éste responde a las preguntas planteadas 
inicialmente en la situación problemática, realizamos particularizaciones, tarea, también, asociada al proceso de modelización.

No podemos descuidar en la enseñanza de la Modelización que los diferentes razonamientos que están implicados en este proceso suelen ser los razonamientos asociados a los esquemas "partes- todo", los razonamientos inductivos y los deductivos.

Señalar, finalmente, que la Competencia Matemática Formal (CMF), que se propone en ELOS, es un instrumento técnico del análisis del contenido matemático que facilita al profesorado de Matemáticas el análisis de los significados atribuidos a los objetos matemáticos desde las perspectivas epistemológicas, semióticas y fenomenológicas, y que resulta de utilidad para caracterizar el dominio de la actividad matemática, de las diferentes tareas que se propongan a los alumnos, y, en consecuencia, para analizar e interpretar las producciones del alumnado en la realización de estas tareas, así como para analizar los errores y hacer conjeturas sobre las dificultades, obstáculos y origen de dichos errores, tanto desde el punto de vista de la docencia como del de la investigación.

Asimismo, las diferentes técnicas de análisis utilizadas son de gran utilidad. Por ejemplo, el análisis del contenido matemático implícito en la pregunta del cuestionario, realizado a priori, nos permite estudiar los diferentes significados que los estudiantes muestran en su resolución. En el análisis a posteriori, el uso de los esquemas de análisis, descritos anteriormente, ha resultado útil para analizar los errores cometidos en la mayoría de las preguntas, y las redes sistémicas han sido un complemento adecuado para ayudar a ver cuáles son los razonamientos seguidos en preguntas abiertas.

En cuanto al Análisis Didáctico, utilizado en este trabajo, en relación a las Dificultades y errores, podemos señalar en lo referente al origen de los errores, que, en general, de los tres orígenes distintos: actitudes afectivas, ausencia de sentido y obstáculo, en el caso de la Modelización, el origen más frecuente es la ausencia de sentido y que éste se manifiesta en dos ámbitos diferenciados, en uno, viene ocasionado por aspectos que han quedado sin resolver en la Aritmética o la Geometría, y en otro, la ausencia de sentido se ha producido mayoritariamente por las características propias del lenguaje algebraico, en los procesos de Sustitución Formal y Generalización. Es fundamental que los alumnos controlen el proceso de Sustitución Formal, implícito en todo proceso de Modelización, y el de Generalización Algebraica, como condición necesaria para la enseñanza competencial de la Modelización.

En relación con las actitudes afectivas, debemos buscar las razones por las que los alumnos no han respondido algunas preguntas. En muchas ocasiones los bloqueos ocasionados por las actitudes son la principal causa que les lleva al error.

Agradecimientos. Este trabajo ha sido financiado parcialmente por el Proyecto de Investigación EDU2011-29324 de Plan Nacional de Investigación del Ministerio de Ciencia e Innovación de España.

\section{Referencias}

Bliss, J., Monk, M., \& Ogborn, J. (1987). Qualitative data analysis for educational research. USA: Croom Helm. 
Blum, W., Galbraith, P., Henn, H., \& Niss, M. (2007) (Eds). Modelling and Applications in Mathematics Education. The $14^{\text {th }}$ ICMI Study. New York: Springer.

Burkhardt, H. (2006). Modelling in mathematics classrooms: reflections on past developments and the future, Zentralblatt für Didaktik der Mathematik, 38 (2), 178-195.

Chevallard, Y. (1989). Le Passage de l'Arithmetique à l'Algebre dans l'enseignement des Mathématiques au Collége (Deuxième partie). Perspectives curriculaires: la notion de modelisation. Petit X, (19), 43-75.

Freudenthal, H. (1983). Didactical phenomenology of mathematical structures. Dordrecht: Reidel.

Kaiser, G., \& Sriramam, B. (2006). A global survey of international perspectives on modelling in mathematics education. Zentralblatt für Didaktik der Mathematik, 38 (3), 302-310.

Peirce, C. S. (1987). Obra Lógico Semiótica. Madrid: Taurus.

Puig, L. (1997). Análisis fenomenológico. En L. Rico (Coord.), La Educación Matemática en la Enseñanza Secundaria (pp. 61-94). Barcelona: ICE Universitat de Barcelona/Horsori.

Rico, L. (1997). Los organizadores del currículo de matemáticas. En L. Rico (Coord.), La Educación Matemática en la Enseñanza Secundaria (pp. 39-59). Barcelona: ICE Universitat de Barcelona/Horsori.

Rico, L., \& Fernández-Cano, A. (2013). Análisis didáctico y metodología de investigación. En L. Rico; J. L. Lupiáñez, \& M. Molina (Eds.). Análisis Didáctico en Educación Matemática. Metodología de investigación, formación de profesores e innovación curricular. (pp. 1-22). Granada: Comares.

Rico L., Lupiáñez, J. L., \& Molina, M. (2013) (Eds.). Análisis Didáctico en Educación Matemática. Metodología de investigación, formación de profesores e innovación curricular. Granada: Comares.

Ruano, R., \& Socas, M. M. (2001). Habilidades cognitivas en relación con la Sustitución Formal, la Generalización y la Modelización que presentan los alumnos de $4 .^{\circ}$ de ESO. En M. M. Socas, M. Camacho, \& A. Morales (Eds.). Formación del profesorado e investigación en Educación Matemática III, pp. 239265.

Socas, M. M. (1997). Dificultades, obstáculos y errores en el aprendizaje de las Matemáticas en la Educación Secundaria. (Cap. V, pp. 125-154). En L. Rico (Coord.), La Educación Matemática en la Enseñanza Secundaria. Barcelona: ICE Universitat de Barcelona/Horsori.

Socas, M. M. (2007). Dificultades y errores en el aprendizaje de las Matemáticas. Análisis desde el Enfoque Lógico Semiótico. Investigación en Educación Matemática XI, pp. 19-52.

Socas, M. M. (2010). Competencia Matemática Formal. Un ejemplo: El Álgebra escolar. Formación del Profesorado e Investigación en Educación Matemática, 10, 9-33.

Socas, M. M. (2012). El análisis del contenido matemático en el Enfoque Lógico 
Semiótico (ELOS). Aplicaciones a la investigación y al desarrollo curricular. En D. Arnau, J. L. Lupiáñez, \& A. Maz (Eds.), Investigaciones en Pensamiento Numérico y Algebraico e Historia de la Matemática y Educación Matemática (pp. 1-22). Valencia: Departamento de Didáctica de la Matemática de Universitat de Valencia y SEIEM.

Socas, M. M., Camacho, M., \& Hernández, J. (2013). Una propuesta formativa de profesores de Matemáticas en la educación obligatoria fundamentada en el Análisis Didáctico desde el Enfoque Lógico Semiótico (ELOS). En L. Rico, J. L. Lupiáñez, \& M. Molina (Eds.). Análisis Didáctico en Educación Matemática. Metodología de investigación, formación de profesores e innovación curricular. (pp. 59-77). Granada: Comares.

\section{Referencias de los autores}

Martín M. Socas, Universidad de La Laguna (España).msocas@ull.edu.es

Raquel Mª Ruano, Universidad de La Laguna (España).rrb75@msn.com

Josefa Hernández, Universidad de La Laguna (España).jhdezd@ull.edu.es 


\title{
A Didactic Analysis of the Mathematical Modelling Process in Secondary School Students
}

\author{
Martín M. Socas, Universidad de La Laguna (España) \\ Raquel M. ${ }^{a}$ Ruano, Universidad de La Laguna (España) \\ Josefa Hernández, Universidad de La Laguna (España)
}

\begin{abstract}
This work didactically analyzes the mathematical modelling process in terms of the difficulties and errors of a group of 60 secondary school students $\left(4^{\text {th }}\right.$ and $5^{\text {th }}$ year of secondary education) from the island of Tenerife.

The mathematical modelling process is discussed in terms of the culture of mathematics and mathematical research as a starting point to study it didactically. In order to do this, there is a description of the basic assumptions of the Semiotic Logical Approach (SLA) (Socas, 2007) and of the models used in the research: Didactic Analysis and Formal Mathematics Competence.

Two problem situations concerning the modelling process are considered, and these are analyzed from the competence point of view using the Formal Mathematical Competence model (FMC), i.e., from the epistemological, semiotic and phenomenological perspective proposed in the Approach (SLA), which facilitates the analysis of the difficulties and errors of the students, in terms of the established Didactic Analysis, as well as the relationships established with the Generalization and Formal Substitution mathematical processes, and the structures and operations involved in the process (phenomenology of the modelling).

The difficulties and mistakes observed in these students in the modelling process are used to identify and organize the errors so that their origin can be conjectured on.

Didactic Analysis is a priori a teaching tool for understanding and developing the organization of the teaching of mathematics. The different mathematical objects which appear in the modelling process should be considered in the organization and development of the teaching and learning of modelling in secondary education. Therefore, the conclusion connects the pupils' difficulties and mistakes with the organization of the teaching of modelling in secondary education, showing, in particular, the role played by Generalization and Formal Substitution in this process, as well as the structures and mathematical operations involved in the process itself.
\end{abstract}

